\author{
Joanna Popielska-Grzybowska (D) \\ Academia Polaca das Ciências \\ CHAM, FCSH/NOVA-Uac \\ jpopielska@iksio.pan.pl
}

\title{
A visão linguística do Além egípcio antigo na tradução para português e inglês ${ }^{1}$
}

\begin{abstract}
Resumo:
A visão linguística do Além no pensamento religioso dos egípcios antigos distingue-se claramente das crenças dos outros povos antigos. A autora do presente artigo pretende analisá-la numa contextualização dos Textos das Pirâmides, citando as traduções das fórmulas diagnosticadas. Analisará a visão linguística na tradução do egípcio hieroglífico para o português, fazendo-se as comparações das traduções das fórmulas para o inglês e os seus obstáculos ou dificuldades nessa tradução.

Usando métodos de análise da linguagem egípcia, compreenderemos melhor o modo de pensar dos egípcios. O método da "visão linguística do mundo" ("linguistic worldview"), isto é, a interpretação verbalizada da realidade, desenvolvida por Jerzy Bartmiński, seus alunos e colaboradores, pode ser excecionalmente útil. A análise linguística minuciosa e profunda dos fragmentos dos textos religiosos
\end{abstract}

${ }^{1}$ Desejo exprimir o meu agradecimento à Comissão Organizadora das $6 a s$ Jornadas de Língua Portuguesa e Culturas Lusófonas da Europa Central e de Leste, que foi muito estimulante para o meu trabalho. Agradeço muito à Senhora Professora Maria Helena Trindade Lopes pela colaboração frutífera e pelo estímulo científico, que me oferece em cada nossa conversa. $\mathrm{O}$ meu muito obrigado ao Senhor Miguel Ângelo Lobo Gonçalves pela ajuda na correção da língua portuguesa deste artigo. 
mais antigos do mundo, na tradução para as línguas contemporâneas, desenvolve uma discussão científica mais completa.

Palavras-chave: Antigo Egito, Textos das Pirâmides, religião antiga, visão linguística, Além egípcio

\section{Abstract: \\ The Linguistic View of the Ancient Egyptian Beyond in Translation into Portuguese and English}

The linguistic worldview of the Beyond in the religious thought of the ancient Egyptians is clearly distinguished from the beliefs of other ancient nations. The author of the paper intends to analyse it as contextualised in the Pyramid Texts, based on her translations of the diagnostic spells. This is an analysis of the linguistic view in the translation from the hieroglyphic Egyptian into Portuguese, making some comparisons of the translations of the spells into English. Some obstacles or difficulties in this translation are evinced.

Using methods of analysis of the Egyptian language, we may better understand the way of thinking of the Egyptians. The linguistic worldview method, namely the verbalised interpretation of reality which was developed by J. Bartmiński, his colleagues and collaborators, can be exceptionally useful. By means of an in-depth linguistic analysis of the fragments of the world's oldest religious texts translated into contemporary languages, one can develop a much more complete scholarly discussion.

Keywords: Ancient Egypt, Pyramid Texts, ancient religion, linguistic worldview, Egyptian Beyond

A tradução do egípcio antigo para as línguas contemporâneas é, só por si, um grande desafio. Porém, enfrentamos uma maior dificuldade quando assumimos a tarefa de traduzir um texto de uma língua antiga e morta para uma outra língua que aprendemos, no caso contemporânea e, logicamente, estrangeira.

Para o conseguir, a análise da linguagem, fraseologia, gramática, etc, tem de depender plenamente do modo como se faz a tradução para qualquer língua contemporânea, aplicando os métodos da linguística comparativa. Obviamente que não é possível conhecer ou saber na íntegra o conceito linguístico egípcio, porque o período de tempo e cultura em que vivemos é completamente diferente, onde pensamos e nos 
exprimimos também de modo diferente. Podemos usar, no entanto, o método de "visão linguística do mundo" ("linguistic worldview"), muito desenvolvido durante as últimas décadas por J. Bartmiński, pelos seus colaboradores e colegas (Tokarski, 2014: 39-66, 303-324; Underhill, 2013: 3-143 e muitos outros), para atingirmos uma melhor compreensão do modo de pensar dos egípcios, neste caso o método adaptado pela autora deste artigo para os estudos dos textos religiosos antigos (Popielska-Grzybowska, 2011: 680-693; 2016: 283-294; 2017: 877-887; 2018: 43-50). Estudar "visão linguística do mundo" é estudar "cultura em língua", ou seja, é a interpretação verbalizada da realidade (Bartmiński, 2009: 11-21; 2012). A análise linguística minuciosa dos fragmentos dos textos religiosos permite desenvolver uma argumentação muito mais completa do que outros estudos realizados, sendo relevante à observação das palavras usadas pelos egípcios para exprimir as suas crenças relacionadas com o Além. Este tipo de exame também tem em conta analisar profundamente todo o contexto textual e cultural (o chamado "Sitz im Leben"/“life context"), a gramática, as mensagens verbais, as palavras, as frases e a própria língua dos textos religiosos. Indubitavelmente, para se poder alcançar não só as traduções corretas para a língua portuguesa, como também o significado do que representa o verdadeiro valor linguístico, é indispensável a consulta das examinações da linguística portuguesa (Mira Mateus, Villalva, 2007).

Os Textos das Pirâmides são o mais velho "livro" religioso do mundo, descobertos por Gaston Maspero nos anos 1880-1881 nos túmulos dos reis das V e VI dinastias (Maspero, 1894) do Antigo Império (2380-2180 a.C., após: Schneider 1996: 494).

Os textos foram escritos em forma de colunas verticais nas paredes das pirâmides. Em algumas pirâmides os sinais gravados - hieroglíficos - estão enchidos com uma espécie de massa semelhante ao vidro, que inicialmente era de cor azul, mas que depois mudou para verde. Para obter aquela cor, os egípcios provavelmente usaram o chamado "azul egípcio" ("Egyptian blue") conhecido como silicato de cobre e cálcio $\left(\mathrm{CaCuSi}_{4} \mathrm{O}_{10}\right.$ ou $\left.\mathrm{CaO} \cdot \mathrm{CuO} \cdot 4 \mathrm{SiO}_{2}\right)$. É considerado como 
o primeiro pigmento sintético. Presume-se que os sinais azuis tinham que imitar o lápis-lazúli ou lazulita.

Muitas das frases nos Textos das Pirâmides podem ser traduzidas até 15 modos diferentes e nem sempre temos a certeza de qual é o correto. Não existia no egípcio antigo daquela época o conceito gramatical de tempo verbal. Além disso, a língua dos Textos das Pirâmides, em que os textos foram escritos nas paredes das pirâmides dos reis, é díspar em relação à língua oficial do Império Antigo, e claramente, de todas as etapas da língua egípcia que surgiram mais tarde. Como não existem muitos estudos da língua e linguagem egípcias em português (Trindade Lopes 1998: 107-110; 2003; Gurgel Pereira 2014; 2016), e nenhuma análise dos Textos das Pirâmides, além dessas da autora, esta constitui um dos primeiros estudos nesta área.

É bastante claro que os Textos das Pirâmides foram compostos e escritos nas paredes dos túmulos para benefício e proveito do rei. Sendo assim, eles permitem uma descrição da viagem do faraó para o céu e da sua realidade celestial. O percurso é quase sempre ascendente, é no sentido de se conservar entre as estrelas, entre os deuses e os seres primordiais.

O Além egípcio foi um lugar situado no céu entre as estrelas imperecíveis ou indestrutíveis. Porque o Além-mundo era predestinado como sendo o lugar de destino do rei, ninguém nele podia impedir a ida e a permanência do faraó. Para a concretização deste objetivo, todas as forças hostis e todos aqueles que dissessem algo contra nome do faraó, teriam de ser impedidos nas suas más intenções pelos deuses.

A julgar pelas informações contextualizadas dos Textos das Pirâmides, o outro mundo era para os egípcios um espaço aquático com as maiores e as mais pequenas ilhas da terra nele existentes. A principal característica do céu $p t$ é a água $m w$. Uma passagem refere-se diretamente às "águas... que estão no céu” (TP fórmula 685 § 2063a). A natureza aquosa está implícita em verbos que são usados para expressar a jornada ou viagem através do céu. Temos os exemplo de: $n m j$ atravessar (com um determinativo de barco); hnnz, viagar; $\underline{h} n j$, remar seu barco; e $\underline{d} 3 j$, cruzar ou atravessar. A descrição mais adequada do céu está formulada por James P. Allen: 
indications from the Pyramid Texts suggest an early image of the celestial domain as an expanse (pd wwt) of water (bj3, kbhw) above the Earth (hrt), whose shores (jdbw pt) consist of marshland (sht j3rw, sht htp) with canals (mrw ptrw(ptrtj)) and lakes (šjw), bordered perhaps by desert (w`rt, jzkn). (Allen, 1989: 9)

Portanto, o defunto primeiro teve de subir para atingir o céu, em que todos os deuses e seres o ajudaram:

$\S 604 \mathrm{a} w \underline{d} . n \mathrm{nw} \mathrm{ttj} n \mathrm{tm}$

$\S 604 \mathrm{~b} w \underline{d} . n \mathrm{pg} 3 \mathrm{ttj} n \check{s} w$

$\S 604 \mathrm{c} \underline{d} .(f) j . w n . t j ~ \Im 3 w j$ pt jpf n ttj hr rmt

$\S 604 d$ nj rn.sn js

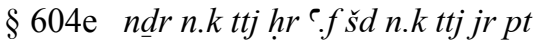

$\S 604 \mathrm{f}$ jm.f $m t j \mathrm{r} t 3 \mathrm{~mm} \mathrm{rm} \underline{\mathrm{t}}$

Nu tinha mandado Téti a Atum. Aquele-dos-braços-abertos tinha mandado Téti a Shu para que ele pudesse abrir aquela porta do céu para Téti, que está entre o povo sem nome: 'Leva Téti pelo seu braço, leva Téti para o céu para que ele não morra na terra entre os seres humanos. ( $T P$ fórmula 361 (T 183))

$\mathrm{Nu}$ has commended Teti to Atum, This-who-is-open-armed has commended Teti to Shu, that he might have opened yonder door of the sky to Teti, now among unidentified people, (saying): "Take Teti by his arm, take Teti to the sky, that he may not die on earth among people. ${ }^{2}$

Por respeito ao limite permitido da dimensão desta publicação, vamo-nos concentrar apenas em alguns pormenores significativos e relevantes ao assunto.

De seguida, o rei viajou de barcaça com o deus sol Rá. Esta viagem auxiliou na revivificação do faraó, cíclica e diariamente repetida:

2 Todos os fragmentos dos Textos das Pirâmides são traduzidos do egípcio antigo para português e inglês pela autora do artigo, baseando-se na publicação do texto hieroglífico publicado por Sethe (Sethe, 1908-1922) e adições baseadas em descobertas posteriores. 
$\S 2172 \mathrm{c} h 3 j j \mathrm{NN} m$ wj3 $r^{\mathrm{c}} \mathrm{h} r \mathrm{j} d b w n w \mathrm{~s} j \mathrm{j}-n-h \mathrm{~h} 3$

$\S 2173 \mathrm{a} \underline{h}$ n.t $N N j n j . h m w-w r \underline{d}$

$\S 2173 \mathrm{~b} w \underline{d} N N m d w n j . h m w-s k$

O rei deve ir a bordo do barco como Rá nas margens do enrolamento. Hidrovia, o rei pode ser remado pelas Estrelas que não conhecem cansaço e pode dar ordens para as Estrelas Imperecíveis... (TP fórmula $697 \S \S 2172 \mathrm{c}-73 \mathrm{~b}$ )

The King shall go aboard the boat like Ra on the banks of the Winding Waterway, the King can be rowed by the Unwearying Stars and can give orders to the Imperishable Stars...

Consequentemente, o faraó nunca está morto, nunca morre como uma pessoa comum, apenas está a passar por várias metamorfoses:

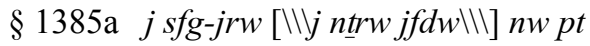

$\S 1385 \mathrm{~b}$ nj mt.n js jt wsjr NN mtt

$\S 1385 \mathrm{c}$ 3h.n jt pw wsjr NN 3ht

Ó vós cujas formas são misteriosas [e vós quatro deuses] do céu, meu pai Osíris o rei não morreu da morte, porque meu pai Osíris o rei tornou-se espírito no horizonte. (TP fórmula $556 \S 1385$ (P 529))

O you whose form is mysterious [and you four gods] of the sky, my father Osiris the King has not died the death, for my father Osiris the King became spirit in the horizon.

Todas as metamorfoses servem para atingir o céu e a última etapa da metamorfose, nomeadamente, tornar-se um só ser com o deus criador:

§ 134a h3 wnjs nj šm.n.kjs mt.tj šm.n.k `nh.t

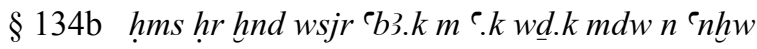

$\S 134 \mathrm{c}$ mks nhbt.km $\mathrm{c} . k w \underline{d} m d w n$ s $t 3 w$ swt

$\S 135 \mathrm{a}$ 'wj.k $\mathrm{m}$ tm rmnwj.k $\mathrm{m} \mathrm{tm}$ ht.k $\mathrm{m}$ tm s3.k $\mathrm{m} \mathrm{tm}$

$\S 135 \mathrm{~b}$ ph.k $\mathrm{m}$ tm rdwj.k $m$ tm hr.k $m z 3 b$

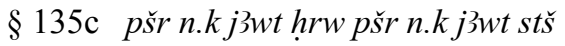


Ó Unis, não é morto, mas vivo, que tens partido,

Toma assento no trono de Osíris!

O teu cetro $[b 3$ está na tua mão para que dês as ordens aos vivos, Mekes e o teu cetro nehbet estão na tua mão, então dá as ordens àqueles-cujos-lugares-estão-ocultos.

Os teus braços são esses de Atum, os teus ombros são esses de Atum, A tua barriga é essa de Atum, as tuas costas são essas de Atum, As tuas nádegas são essas de Atum, as tuas pernas são essas de Atum. A tua cara é essa do Chacal.

Que as colinas de Horus te sirvam e que as colinas de Seth te sirvam. (TP fórmula 213 (W 146))

O Unis, it is not dead but alive that you have gone away.

Sit upon the throne of Osiris!

Your sceptre $a b a$ is in your hand, may you give orders to the living. mekes and your sceptre nekhebet are in your hand, so give orders to those-whose-seats-are-hidden.

Your arms are those of Atum, your shoulders are those of Atum,

Your belly is that of Atum, your back is that of Atum,

Your hind-parts are those of Atum, your legs are those of Atum,

Your face is that of a Jackal.

Let the mounds of Horus serve you, and let the mounds of Seth serve you.

A fim de obter a identificação discutida, foi usada uma forma gramatical, a chamada " $m$ de predicação". Quando os egípcios usaram esta preposição nesta função, pretenderam a identificação de dois seres ou objetos, e não a sua comparação, porquanto, se tivessem desejado descrever semelhante, teriam usado outra preposição, ou seja, $m r$. Para traduzir estas preposições para inglês, podemos diferenciar: $m$ é "as", e $m r$ é "like"; contudo, em português não existem duas formas diversas, sendo que a perceção da criação pelo poder da palavra no egípcio tem de ser explicada de modo descritivo em português. Os egípcios acreditavam que as palavras escritas e depois pronunciadas em alta voz criavam uma realidade, isto é, o descrito nas palavras efetivamente acontecia. 


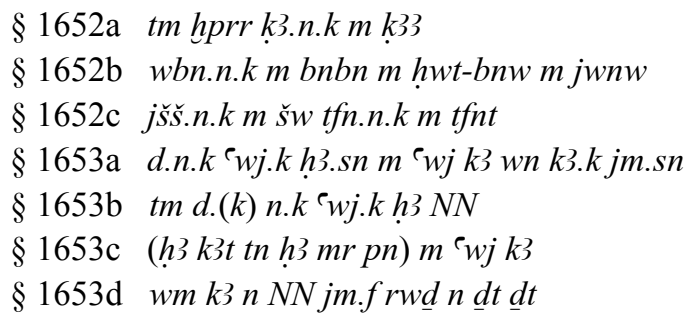

Atum Kheprer/Atum-que-está-a-formar-se! Tu ficaste elevado como a colina, tu apareceste como benben na Residência do pássaro Benu em Junu. Tu sopraste Shu e expeliste Tefnut. Tu puseste os teus braços ao redor deles assim para que o teu $k a$ pudesse estar neles.

Ó Atum, coloca os seus braços sobre o rei, sobre esta construção, e sobre esta pirâmide como os braços de $k a$, para que a essência do rei possa estar nele, durando para todo o sempre. (TP fórmula 600 (N 359))

Atum Kheprer (who-is-coming-into-being)! You became high, as the hill; you rose as the benben in the Enclosure of the Benu-bird in Junu. You sneezed Shu and spat out Tefnut. You put your arms around them as $k a-$ -arms so that your ka might be in them.

O Atum, place your arms about the King, about this construction, and about this pyramid as the arms of $k a$, that the king's essence may be in it, enduring for ever and ever.

Do exemplo citado, existe uma distinção evidente e de fácil perceção entre a intenção egípcia de identificar e a de comparar. Por conseguinte, a identificação significou criar a desejada realidade religiosa, em particular no Além-mundo para o bem do faraó.

Desta maneira, e sendo o criador que sempre vive no Além, o rei que deixou a terra para ir para o céu, torna-se o pleno de cada deus e só nestas condições governa o universo e sobrevive ao fim do mundo, sendo o criador que tem tudo e todos em si, cada elemento da criação. Assim, o rei ficou completo física e psicologicamente, passando as partes do seu corpo a ser as dos diversos deuses. Por causa da identificação com os deuses, o faraó tornou-se a essência $k a$ para viver eternamente: 
$\S 147 \mathrm{~b}$ tn kw jn.sn m rn.kn ntr hpr.kj.tm.t (m) ntr $n b$

$\S 148 \mathrm{a}$ tp.k $m$ hrw d3t j.hmm-sk

$\S 148 \mathrm{~b}$ mhnt.k m hntj-jrtj j.hm-sk

§148c msdrwj.kz3tj tm j.hnm-skjrtj.kz3tj tm j.hmm-sk

$\S 148 \mathrm{~d}$ fnd.k $m$ z3bj.hm-sk jbhw.k spdwj.hm-sk

$\S 149 \mathrm{a}$ ‘wj.k hp dw3-mjwt.f dbh.kpr.k r pt prr.k

$\S 149 \mathrm{~b}$ rdwj.kjmst kbh-snw.f dbh.k h3.k jr nwt h33.k

$\S 149 \mathrm{c}$ 'wt.kz3tj tm j.hnm-sk

$\S 149 \mathrm{~d} n j$ sk.k nj sk k3.ktwt $k$ 3

Tu estás distinguido - dizem eles, no teu nome do deus, fica completo de/ como (sendo) cada deus (Popielska-Grzybowska, 2001: 120-123):

A tua cabeça é a de Horus da Duat, Ó Indestrutível!

A tua cara é a do Khentj-jrtj, Ó Indestrutível!

As tuas orelhas são as dos Gémeos de Atum, Ó Indestrutível!

Os teus olhos são os dos Gémeos de Atum, Ó Indestrutível!

O teu nariz é o do Chacal, Ó Indestrutível!

Os teus dentes são os do Sopdu, Ó Indestrutível!

Os teus braços são os do Hep e Duamutef, Ó Indestrutível!

Dos quais tu precisas para ascender ao céu - e tu ascenderás.

As tuas pernas são as de Jmsetj e Kebehsenuf,

Das quais tu precisas para descer ao céu baixo - e tu descerás.

Todos os teus membros são os dos Gémeos de Atum, Ó Indestrutível!

Tu não ficarás aniquilado e o teu $k a$ não ficará aniquilado - tu és $k a$ !

(TP fórmula $215 \S 147 \mathrm{~b}-149 \mathrm{~d}$ )

You are distinguished - say they, in your name of god become complete of/as every god: Your head is that of Horus of the Duat, O Imperishable!

Your face is that of Khentj-jrtj, O Imperishable!

Your ears are those of the Twins of Atum, O Imperishable!

Your eyes are those of the Twins of Atum, O Imperishable!

Your nose is that of the Jackal, O Imperishable!

Your teeth are those of Sopdu, O Imperishable!

Your arms are those of Hep and Duamutef, which you need to ascend to the sky - and you shall ascend.

Your legs are Jmsetj and Kebehsenuf, 
which you need to descend to the lower sky - and you shall descend.

All your members are the Twins of Atum, O Imperishable!

You shall not perish and your $k a$ shall not perish - you are $k a$.

Seja na língua portuguesa ou inglesa, em nenhuma delas existe uma ligação entre a preposição completo/complete com a preposição 'de' ou 'of', mas na língua egípcia é exigida esta expressão para conseguirmos o sentido verdadeiro da mensagem. Por isso, precisamos de usar: "completo de cada deus" e "complete of every god". É assim, porque a tal plenitude do rei como o criador vem DE cada deus. Porém, outra possibilidade é compreender o " $m$ " como a palavra usada para unir os substantivos, isto é, "como". Em inglês o tradutor tem à sua disposição uma palavra para exprimir comparação, nomeadamente: "like" (something is like something) e outra para exprimir identificação, ou seja, "as" (you as someone did something). Por exemplo: "I as a mother am caring / Eu como mãe sou carinhosa", mas "I as a teacher am demanding / Eu como professora sou exigente”. Esta identificação é usada para assumir as identidades diferentes e realizar os diversos papéis. Esta foi a mesma função na língua egípcia para amparar as metamorfoses do faraó, para ele se tornar o criador na última transformação ou metamorfose no Além. Assim, ele, o faraó-criador, pode governar todo o universo - os vivos e os mortos.

Os egípcios acreditavam no poder criador das palavras escritas e pronunciadas. Consequentemente, o que foi escrito recebeu a sua forma real e começou a funcionar "ao vivo".

Podíamos, evidentemente, multiplicar os exemplos relevantes ao assunto. Porém, este artigo é apenas uma introdução e um princípio de pesquisas linguísticas nos Textos das Pirâmides em português. Por isso, esperamos que os excertos citados tenham aproximado a excecionalidade da língua religiosa egípcia e a sua linguagem no seu serviço. Outrossim, as comparações dos textos egípcios traduzidos para inglês e português apresentam as possibilidades que a língua portuguesa oferece aos estudiosos dos textos religiosos do país dos faraós. 


\section{Referências bibliográficas}

ALLEN, J. P. (1989), "The Cosmology of the Pyramid Texts" em: Simpson, W. E. (ed.), Religion and Philosophy in Ancient Egypt, YES 3, New Haven, pp. 1-28.

BARTMIŃSKI, J. (2009), Językowe podstawy obrazu świata, Lublin.

BARTMIŃSKI, J. (2012), Aspects of Cognitive Ethnolinguistics, equinox, London.

MASPERO, G. (1894), Les Inscriptions des Pyramides de Saqqarah, Bouillon, Paris.

MIRA MATEUS, M. H., VILLALVA, A. (2007), Linguística, Editorial Caminho, Lisboa.

TRINDADE LOPES, M. H. (1998), "Eu sou o ontem, o hoje e o amanhã" (Livro dos Mortos do antigo Egipto, cap. 6, Revista da Faculdade de Ciências Sociais e Humanas, 12, Lisboa, pp. 107-110.

TRINDADE LOPES, M. H. (2003), Estudos de Egiptologia, Associação Portuguesa de Egiptologia, Lisboa.

GURGEL PEREIRA, R. G. (2014, 2016), Egípcio hieroglifico. Para o estudo do estágio inicial da língua egípcia (de 3000 a 1300 a.C.), Chiado Editora, Lisboa.

POPIELSKA-GRZYBOWSKA, J. (2001), "Atum in the Pyramid Texts" em: Popielska-Grzybowska, J. (ed.), Proceedings of the First Central European Conference of Young Egyptologists, Egypt 1999: Perspectives of Research, Warsaw 7-9 June 1999, Światowit Supplement Series E: Egyptology, vol. I, WES, vol. III, Wydawnictwo IA UW, Warsaw, pp. 115-129.

POPIELSKA-GRZYBOWSKA, J. (2011), "Religious Reality Creation through Language in the Old Kingdom Religious Texts" em: Bárta, M., Coppens, F., Krejči, J. (ed.) Abusir and Saqqara in the Year 2010, Czech Institute of Egyptology Press, Prague, pp. 680-693; bibliografia abrangente pp. 823-904.

POPIELSKA-GRZYBOWSKA, J. (2016), “A colaboração científica portuguesa-polaca na Egiptologia e as traduções do egípcio antigo para português" em: Hlibowicka-Węglarz, B., Wiśniewska, J., Jabłonka, E. (ed.), Lingua Portuguesa. Unidade na Diversidade, Lublin 2016, pp. 283-294. 
POPIELSKA-GRZYBOWSKA, J. (2017), “A visão linguística do criador egípcio nos Textos das Pirâmides - algumas palavras de introdução" em: Bernal, L., Pérez Largacha, A., Vivas Sáinz, I. (ed.), V Congresso Ibérico de Egiptologia, Ediciones de Castilla-La Mancha, Castilla-La Mancha (e-publication), pp. 877-887.

POPIELSKA-GRZYBOWSKA, J. (2018), “Antigas Cartas egípcias aos Mortos. Cartas de negócios muito peculiares?”, $C E M$, 8, Porto, pp. 43-50.

SCHNEIDER, T. von (1996), Lexikon der Pharaonen, dtv, München.

SETHE, K. (1908-1922), Die altägyptischen Pyramidentexte, Hinrichs, J. C., Leipzig, vols. 1-4.

TOKARSKI, R. (2014), Światy za słowami. Wykłady z semantyki leksykalnej, Wydawnictwo Uniwersytetu Marii Curie-Skłodowskiej, Lublin.

UNDERHILL, J.W. (2013), Humboldt, Worldview and Language, Edinburgh University Press, Edinburgh. 